\title{
Consistent Asymptotic Expansion of Mott's Solution for Oxide Growth
}

\author{
Matthew R. Sears and Wayne M. Saslow \\ Department of Physics, Texas A\&M University, \\ College Station, Texas 77840-4242
}

\begin{abstract}
Many relatively thick metal oxide films grow according to what is called the parabolic law $L=$ $\sqrt{2 A} t+\ldots$ Mott explained this for monovalent carriers by assuming that monovalent ions and electrons are the bulk charge carriers, and that their number fluxes vary as $t^{-1 / 2}$ at sufficiently long $t$. In this theory no charge is present in the bulk, and surface charges were not discussed. However, it can be analyzed in terms of a discharging capacitor, with the oxide surfaces as the plates. The theory is inconsistent because the field decreases, corresponding to discharge, but there is no net current to cause discharge. The present work, which also includes non-monovalent carriers, systematically extends the theory and obtains the discharge current. Because the Planck-Nernst equations are nonlinear (although Gauss's Law and the continuity equations are linear) this leads to a systematic order-by-order expansion in powers of $t^{-1 / 2}$ for the number currents, concentrations, and electric field during oxide growth. At higher order the bulk develops a non-zero charge density, with a corresponding non-uniform net current, and there are corrections to the electric field and the ion currents. The second order correction to ion current implies a logarithmic term in the thickness of the oxide layer: $L=\sqrt{2 A} t+B \ln t+\ldots$ It would be of interest to verify this result with high-precision measurements.
\end{abstract}

\section{INTRODUCTION}

From the late 1930s to the late 1940s, N.F. Mott considered $^{1+3]}$ the implications of experimental results 4 for oxide growth. (For more of a review, see Ref 5.) Under certain conditions (particularly high temperature), many metals develop ${ }^{4}$ a layer of oxide at a parabolic rate on surfaces exposed to gas containing oxygen, according to

$$
L^{2}=2 A t
$$

where $L$ is the thickness of the oxide, $t$ is time, and $A$ is a constant. The rate of growth is thus

$$
\frac{d L}{d t}=\sqrt{\frac{A}{2}} t^{-1 / 2} .
$$

This result may be thought of as representing the longtime asymptotic limit. For specificity, we assume that the metal M fills $x<0$, that oxide MO fills $0<x<L$, and that oxygen gas $\mathrm{O}$ fills $L<x$. This means we employ a moving coordinate system where $x=0$ represents the $\mathrm{M} / \mathrm{MO}$ interface, and $x=L(t)$ represents the $\mathrm{MO} / \mathrm{O}$ interface.

A field and fluxes that vary as $t^{-1 / 2}$ are expected on the basis of a gradient of concentration, with the values of the carrier concentrations pinned by the two surfaces and the length $L$ determining the gradient. 4 That is, $d L / d t \sim 1 / L$ gives a parabolic law. Wagner obtained a parabolic growth law using the Planck-Nernst equations and some additional assumptions. ${ }^{6}$ Mott obtained a parabolic growth law using a more complete argument ${ }^{2}$ that invokes the Planck-Nernst equations, Gauss's Law, and (implicitly) the continuity equations. In this case one can think of the electrochemical potentials pinned by the two surfaces and $L$ determining the gradient, which then yields the parabolic law.
For electron and ion number currents $\left(j_{a}, j_{b}\right)$ and ion valence $Z=1$, Mott assumed that the total current $J=$ $e\left(j_{b}-j_{a}\right)$ in the oxide is zero, so

$$
j_{a}=j_{b}
$$

Since oxide grows when metal ions reach the oxide/gas interface, the growth rate is

$$
\frac{d L}{d t}=j_{b} \Omega
$$

where $\Omega$ is the volume per metal ion in the newly formed oxide. Comparison with (2) immediately shows that

$$
j_{b} \sim t^{-1 / 2}
$$

for the asymptotic behavior of the ion fluxes. By the Planck-Nernst equations, the electric field $E$ and the ion density gradients $\left(\partial_{x} n_{a}, \partial_{x} n_{b}\right)$ also have the same behavior. Moreover, the quantities $\left(j_{a}, j_{b}, E, \partial_{x} n_{a}, \partial_{x} n_{b}\right)$ are all uniform throughout the oxide.

We assume that the metal and the gas are neutral, so by Gauss's Law the surface charges $(\Sigma(0), \Sigma(L))$ and the electric fields $(E(0), E(L))$ are related by

$$
E(0)=\frac{\Sigma(0)}{\epsilon}, \quad E(L)=-\frac{\Sigma(L)}{\epsilon} .
$$

Moreover, by continuity the assumption that there is charge and current only within the oxide leads to the conditions

$$
J(0)=\frac{d \Sigma(0)}{d t}, \quad J(L)=-\frac{d \Sigma(L)}{d t} .
$$

This model immediately poses the questions of whether Mott's solution is self-consistent, and whether it is the beginning of an asymptotic series in powers of $t^{-1 / 2}$. To answer self-consistency, note that the uniform but 
decreasing-with-time $E \sim t^{-1 / 2}$ leads to no bulk charge and to interfaces with equal and opposite charge, so they behave like a capacitor. Since $E$ decreases with time, so must the charge on the capacitor. However, the model assumes zero current. Hence Mott's solution is not selfconsistent.

Nevertheless we will show, in response to the question about asymptopia, that each of the continuous variables can be expanded in an asymptotic series in $t^{-n / 2}$, where Mott's solution corresponds to $n=1$, and that non-zero current $J$ appears at order $n=3$. We will also show that all of the continuous variables can depend upon position. This means that the bulk can develop a local and total charge density, with the surfaces not having equal and opposite charges, so that the capacitor model holds only to lowest order. It is already known that steady nonequilibrium current flow can cause local charge densities in the bulk! ${ }^{78}$

The fact that an expansion can be made of $j_{b}$ in powers of $t^{-1 / 2}$ leads to

$$
\frac{d L}{d t}=\sqrt{\frac{A}{2}} t^{-1 / 2}+B t^{-1}+\ldots
$$

so that

$$
L=\sqrt{2 A} t^{1 / 2}+B \ln t+\ldots
$$

to higher accuracy than given by the pure parabolic law. This prediction can be subjected to experimental study. In practice, we must assume that

$$
L=\sqrt{2 A} t^{1 / 2}+B \ln t+C+\ldots
$$

because $\ln t$ is of order unity. We have not found data of a high enough precision to verify this $\ln t$ correction.

Section II gives the five fundamental equations for the five continuous variables (two continuity equations, Gauss's Law, and two Planck-Nernst equations), and indicates the expansion in powers of $t^{-1 / 2}$. (The fact that it is such an expansion relates to the leading order term and the fact that the Planck-Nernst equation is a nonlinear function of the density.) Section III gives the $n=1$ solution, which has two interface-reaction-rate-determined integration constants: both $A$ and a constant-in-space density not included in the Mott solution. Section IV presents the results of the $n=2$ solution that gives the first correction to Mott's solution, and shows that there is a non-zero bulk charge density and a corresponding spatial variation to the field. There are four interfacereaction-rate-determined integration constants for $n=2$. All higher-order solutions involve four interface-reactionrate-determined integration constants. Section V considers the oxide thickness growth rate. Section VI provides a summary and conclusions. The Appendices explicitly give the $n=2$ and $n=3$ solutions.

\section{ON TWO-COMPONENT TRANSPORT}

Let the subscripts $a$ and $b$ denote electrons and metal ions respectively. Let $n_{0}$ and $n_{0} / Z$ be the uniform equilibrium concentration of electrons and metal ions respectively, $\left(n_{a}, n_{b}\right)$ be their additional concentrations, $\left(\nu_{a}, \nu_{b}\right)$ be their mobilities, $\left(D_{a}, D_{b}\right)$ be their diffusion coefficients, $\left(q_{a}=-e, \quad q_{b}=Z e\right)$ be their charges, $E$ be the electric field, $k_{B}$ be the Boltzmann constant, $T$ be the temperature, and $x$ be the position.

Note that we use the Einstein Relations to rewrite the mobilities, which can have either sign, in terms of diffusion constants, which are always positive. Since we always consider $Z$ positive, if we want to consider oxygen ions and holes as the carriers, then only the sign of the electric charge $e$ must be changed. For $\mathrm{M}^{3+}$ and $\mathrm{O}^{2-}$, we let $e \rightarrow 2 e$ and $Z=3 / 2$. Therefore our results are quite general.

\section{A. Equations for Two-Component Transport}

We will employ the Einstein Relations

$$
\frac{\nu_{a}}{D_{a}}=\frac{q_{a}}{k_{B} T}, \quad \frac{\nu_{b}}{D_{b}}=\frac{q_{b}}{k_{B} T} .
$$

For electrons and metal ions, $-Z q_{a}=q_{b}$, so

$$
\nu_{b} D_{a}=-Z \nu_{a} D_{b}, \quad-\frac{\nu_{a}}{D_{a}}=\frac{\nu_{b}}{Z D_{b}}=\frac{1}{V_{T}},
$$

where

$$
V_{T}=\frac{k_{B} T}{e}
$$

denotes a thermal voltage. Mott and Cabrera's paper ${ }^{\sqrt{5}}$ used $\left(v_{a}, v_{b}\right)$ to denote electron and ion mobilities, which they may have intended to be $\left(\nu_{a}, \nu_{b}\right)$.

The one-dimensional Planck-Nernst equations for the number flux densities associated with metal ions and electrons are

$$
\begin{aligned}
& j_{a}=\nu_{a}\left(n_{0}+n_{a}\right) E-D_{a} \partial_{x} n_{a}, \\
& j_{b}=\nu_{b}\left(\frac{n_{0}}{Z}+n_{b}\right) E-D_{b} \partial_{x} n_{b} .
\end{aligned}
$$

Rewriting mobilities in terms of diffusion constants using 11,

$$
\begin{aligned}
& j_{a}=-\frac{D_{a}}{V_{T}}\left(n_{0}+n_{a}\right) E-D_{a} \partial_{x} n_{a}, \\
& j_{b}=\frac{D_{b}}{V_{T}}\left(n_{0}+Z n_{b}\right) E-D_{b} \partial_{x} n_{b},
\end{aligned}
$$

We also use the number continuity equations,

$$
\partial_{t} n_{a}+\partial_{x} j_{a}=0, \quad \partial_{t} n_{b}+\partial_{x} j_{b}=0,
$$

and Gauss's Law,

$$
\partial_{x} E=\frac{e}{\epsilon}\left(Z n_{b}-n_{a}\right)
$$


where $e$ is electron charge in Coulombs, and $\epsilon$ is the permittivity of the oxide.

With the charge density and current density defined by

$$
\rho=-e\left(n_{a}-Z n_{b}\right), \quad J=-e\left(j_{a}-Z j_{b}\right),
$$

use of the number continuity equations yields the charge continuity equation

$$
\partial_{t} \rho+\partial_{x} J=0
$$

\section{B. Expansion Notation}

We seek a series solution in powers of $t^{-1 / 2}$ for the electron and ion concentrations (densities) and fluxes, as well as for the electric field. They must satisfy the PlanckNernst equations, the continuity equations, and Gauss's Law. Following Mott, we take the lowest order fluxes and field to vary as $t^{-1 / 2}$. Examining the structure of the Planck-Nernst equation for electrons (16), and inserting terms of order $t^{-1 / 2}$, the nonlinear term $n_{a} E$ will contain terms of order $t^{-1}$. Iteration yields that the series must be in powers of $t^{-n / 2}$ for integer $n$.

We thus make an expansion of the form

$$
\begin{aligned}
& j_{a}=\sum_{n=1} J_{a n} t^{-n / 2}, \quad j_{b}=\sum_{n=1} J_{b n} t^{-n / 2}, \\
& n_{a}=\sum_{n=1} N_{a n} t^{-n / 2}, \quad n_{b}=\sum_{n=1} N_{b n} t^{-n / 2}, \\
& \Sigma^{(0)}=\sum_{n=1} \Sigma_{n}^{(0)} t^{-n / 2}, \quad \Sigma^{(L)}=\sum_{n=1} \Sigma_{n}^{(L)} t^{-n / 2}, \\
& E=\sum_{n=1} E_{n} t^{-n / 2}, \\
& J=\sum_{n=1} J_{n} t^{-n / 2}, \quad \rho=\sum_{n=1} \rho_{n} t^{-n / 2} .
\end{aligned}
$$

Here $J_{a n}, J_{b n}, N_{a n}, N_{b n}, E_{n}, \rho_{n}$, and $J_{n}$ are functions of the position along the direction of growth, $x$. From the above definitions, the dimensionality of $\left(J_{a n}, J_{b n}\right)$ is concentration times velocity times $t^{n / 2}$, the dimensionality of $\left(N_{a n}, N_{b n}\right)$ is concentration times $t^{n / 2}$, the dimensionality of the surface charge density $\left(\Sigma_{n}^{(0)}, \Sigma_{n}^{(L)}\right)$ is charge per area times $t^{n / 2}$, the dimensionality of $E_{n}$ is electric field times $t^{n / 2}$, the dimensionality of $\rho_{n}$ is charge density times $t^{n / 2}$, and the dimensionality of $J_{n}$ is current density times $t^{n / 2}$.

\section{On Specifying Chemical Reaction Rates at Surfaces}

In the presence of a true chemical reaction at a surface there is a single reaction rate, typically specified by a Butler-Volmer relation,, 11 between the fluxes of all of the relevant components. In the present case the fluxes of the carriers are independent of one another, so that there are two statements about carrier fluxes at each surface, for a total of four conditions. With $\tilde{\mu}$ denoting an electrochemical potential, near equilibrium (as we have here, in the asymptotic regime) each flux $j$ will be proportional to its corresponding $\Delta \tilde{\mu}$ across the interface (either $\mathrm{M} / \mathrm{MO}$ or $\mathrm{MO} / \mathrm{O}$ ). The proportionality constant will depend details of the reaction and the baseline properties of the system. That is,

$$
j_{a, b}=G_{a, b} \Delta \tilde{\mu}_{a, b}
$$

at each surface, so there are four $G$ 's. The equation becomes nonlinear far from equilibrium. Thus the $j_{a, b}$ are proportional to a non-equilibrium quantity, which we take to be a field $E_{1}$, as in Ref. 5. All of the unknown integration constants will be linear or higher in $E_{1}$.

We will not attempt to carry this procedure any further. It is sufficient for our purposes to know that this can be done, and that in the present problem there are four constants associated with boundary conditions at the two surfaces for the two carriers. In principle, all of the quantities appearing in the solutions to the transport equations are determined by these surface reaction rates. For a true chemical reaction, which we expect to be described by a Butler-Volmer equation, the fluxes at each surface, because they are related, will be described by only a single independent coefficient $G$. Note also that the Butler-Volmer equation is non-linear, so that the boundary conditions can be nonlinear. Because we do not consider the boundary conditions in detail, we will neglect this possibility.

\section{RELATIONS BETWEEN EXPANSION COEFFICIENTS: ALL $n$}

\section{A. Continuity Relations and Charge Conservation}

The continuity equations imply charge conservation, so we treat them in the same subsection.

The continuity equations (18) yield

$$
\sum_{n=1}\left(\partial_{x} J_{a n}\right) t^{-n / 2}+\sum_{m=1} N_{a m}\left(-\frac{m}{2}\right) t^{-(m+2) / 2}=0,
$$

for subscript $a$, and a similar relation holds for $b$. With $m=(n-2)$, so that $\sum_{m=1} \rightarrow \sum_{n=3}$, comparison of like powers of $t$ yields, for $n=1$ and $n=2$,

$$
\partial_{x} J_{a n}=0, \quad \partial_{x} J_{b n}=0, \quad(n=1,2),
$$

and, for $n \geq 3$,

$$
\begin{aligned}
& \partial_{x} J_{a n}=\left(\frac{n-2}{2}\right) N_{a(n-2)}, \\
& \partial_{x} J_{b n}=\left(\frac{n-2}{2}\right) N_{b(n-2)}, \quad(n \geq 3) .
\end{aligned}
$$


By definition we have

$$
\rho_{n}=e\left(Z N_{b n}-N_{a n}\right), \quad J_{n}=e\left(Z J_{b n}-J_{a n}\right),
$$

and charge conservation for each $n$ is

$$
\partial_{t} \rho_{n}+\partial_{x} J_{n}=0
$$

Charge conservation at each surface yields

$$
\begin{aligned}
& \frac{d \Sigma^{(0)}}{d t}=-\left.J\right|_{x=0}=-\left.e\left(Z j_{b}-j_{a}\right)\right|_{x=0}, \\
& \frac{d \Sigma^{(L)}}{d t}=\left.J\right|_{x=L}=\left.e\left(Z j_{b}-j_{a}\right)\right|_{x=L},
\end{aligned}
$$

so

$$
\begin{aligned}
& \sum_{n=1}\left(-\frac{n}{2}\right) \Sigma_{n}^{(0)} t^{-(n+2) / 2} \\
& =-\left.e \sum_{n=1}\left(Z J_{b n}-J_{a n}\right)\right|_{(x=0)} t^{-n / 2} \\
& \sum_{n=1}\left(-\frac{n}{2}\right) \Sigma_{n}^{(L)} t^{-(n+2) / 2} \\
& =\left.e \sum_{n=1}\left(Z J_{b n}-J_{a n}\right)\right|_{(x=L)} t^{-n / 2} .
\end{aligned}
$$

Comparing powers of $t$ we have, for $n=1$ and $n=2$,

$$
\begin{aligned}
& \left.Z J_{b n}\right|_{(x=0)}=\left.J_{a n}\right|_{(x=0)}, \\
& \left.Z J_{b n}\right|_{(x=L)}=\left.J_{a n}\right|_{(x=L)}, \quad(n=1,2),
\end{aligned}
$$

and, for $n \geq 3$,

$$
\begin{aligned}
& -\left(\frac{n-2}{2}\right) \Sigma_{(n-2)}^{(0)}=-\left.e\left(Z J_{b n}-J_{a n}\right)\right|_{(x=0)}, \\
& -\left(\frac{n-2}{2}\right) \Sigma_{(n-2)}^{(L)}=\left.e\left(Z J_{b n}-J_{a n}\right)\right|_{(x=L)},
\end{aligned}
$$$$
(n \geq 3) \text {. }
$$

Charge conservation over both surface and bulk yields

$$
\begin{aligned}
& \sum_{n=1}\left(\Sigma_{n}^{(0)}+\Sigma_{n}^{(L)}\right) t^{-n / 2} \\
& \quad=e \int_{0}^{L} \sum_{n=1}\left(N_{a n}-Z N_{b n}\right) t^{-n / 2} d x,
\end{aligned}
$$

so that, for each $n$,

$$
\Sigma_{n}^{(0)}+\Sigma_{n}^{(L)}=e \int_{0}^{L}\left(N_{a n}-Z N_{b n}\right) d x .
$$

\section{B. Gauss's Law}

Gauss's Law 19 reads

$$
\sum_{n=1}\left(\partial_{x} E_{n}\right) t^{-n / 2}=\frac{1}{\epsilon}\left(\sum_{n=1} \rho_{n} t^{-n / 2}\right),
$$

so, for each $n$,

$$
\partial_{x} E_{n}=\frac{1}{\epsilon} \rho_{n}
$$

Gauss's Law at the surfaces 6 gives, for each n,

$$
E_{n}(0)=\frac{\Sigma_{n}^{(0)}}{\epsilon}, \quad E_{n}(L)=-\frac{\Sigma_{n}^{(L)}}{\epsilon} .
$$

\section{Planck-Nernst}

The Planck-Nernst equation for species $a, 16$, can be written as

$$
\begin{aligned}
& \sum_{n=1} J_{a n} t^{-n / 2}=-\frac{D_{a}}{V_{T}} n_{0} \sum_{n=1} E_{n} t^{-n / 2} \\
& \quad-\frac{D_{a}}{V_{T}} \sum_{m, n=1} N_{a n} E_{m} t^{-(m+n) / 2}-D_{a} \sum_{n=1}\left(\partial_{x} N_{a}\right) t^{-n / 2} .
\end{aligned}
$$

or,

$$
\begin{aligned}
\sum_{n=1}\left(J_{a n}\right. & \left.+\frac{D_{a}}{V_{T}} n_{0} E_{n}+D_{a} \partial_{x} N_{a n}\right) t^{-n / 2} \\
= & -\frac{D_{a}}{V_{T}} \sum_{m, n=1} N_{a n} E_{m} t^{-(m+n) / 2},
\end{aligned}
$$

with a similar form for species $b$,

$$
\begin{aligned}
\sum_{n=1}\left(J_{b n}\right. & \left.-\frac{D_{b}}{V_{T}} n_{0} E_{n}+D_{b} \partial_{x} N_{b n}\right) t^{-n / 2} \\
= & \frac{Z D_{b}}{V_{T}} \sum_{m, n=1} N_{b n} E_{m} t^{-(m+n) / 2} .
\end{aligned}
$$

By matching coefficients of powers of $t$, we obtain the resultant equations for any $n$.

\section{Solving the Transport Equations}

There are five first-order differential equations for five continuous variables, so there are five integration constants at each order. For $n \geq 3$ the current density $J_{n}$ at $x=L$ is known from the $n-2$ value of surface charge density $\Sigma_{n-2}^{(L)}$. Therefore only four integration constants need be determined. These can be thought of as fixed by the "reaction rates" of each charge carrier at each of the interfaces, which will not be specified.

The cases $n=1$ and $n=2$ are a bit simpler than $n \geq 3$. Nevertheless, for each $n$ our strategy will be the same: (1) from the continuity equations find the ion fluxes $J_{a n}$ and $J_{b n} ;(2)$ from all of the five equations find an equation for $\rho_{n}$ and solve it; (3) use this $\rho_{n}$ in Gauss's Law to find $E_{n} ;(4)$ find $N_{a n}$ and $N_{b n}$ by substitution of $J_{a n}, J_{b n}$ and $E_{n}$ into the Planck-Nernst equations. 


\section{SOLUTION FOR $n=1$}

For $n=1$, the calculations are simple, but illustrate what happens in higher orders. The continuity equations (29) give $J_{a 1}$ and $J_{b 1}$ to be uniform, and charge conservation at each surface $(36)$ gives

$$
J_{a 1}=Z J_{b 1} \text {. }
$$

The Planck-Nernst equations (44) and 450 yield

$$
\begin{aligned}
& J_{a 1}+\frac{D_{a}}{V_{T}} n_{0} E_{1}+D_{a} \partial_{x} N_{a 1}=0, \\
& J_{b 1}-\frac{D_{b}}{V_{T}} n_{0} E_{1}+D_{b} \partial_{x} N_{b 1}=0,
\end{aligned}
$$

where we take $E_{1}$ as a uniform, experimentally determined value.

From the uniformity of $E_{1}$,

$$
\partial_{x} E_{1}=0
$$

so that Gauss's Law yields

$$
\rho_{1}=0, \quad N_{a 1}=Z N_{b 1} .
$$

Substitution of fluxes from (46) and concentrations from (50) into the Planck-Nernst equations gives

$$
\begin{aligned}
& J_{a 1}+\frac{D_{a}}{V_{T}} n_{0} E_{1}+D_{a} \partial_{x} N_{a 1}=0, \\
& \frac{J_{a 1}}{Z}-\frac{D_{b}}{V_{T}} n_{0} E_{1}+D_{b} \partial_{x} N_{b 1}=0 .
\end{aligned}
$$

Thus, with 50), we have

$$
\begin{aligned}
& J_{a 1}=Z J_{b 1}=-(1+Z) \frac{D_{a} D_{b}}{D_{b}-D_{a}} \frac{n_{0} E_{1}}{V_{T}}, \\
& N_{a 1}=Z N_{b 1}=\frac{H n_{0} E_{1}}{V_{T}} x+M_{1}
\end{aligned}
$$

here,

$$
H=\left(\frac{D_{a}+Z D_{b}}{D_{b}-D_{a}}\right)
$$

is dimensionless, and $M_{1}$ is a constant of integration (units of concentration times $\mathrm{s}^{1 / 2}$ ) determined by the surface reaction rates (and thus linear in $E_{1}$ ). (Recall that $\left(J_{a 1}, N_{a 1}\right)$ are first order coefficients, which must be multiplied by $t^{-1 / 2}$ in order to find the flux and number densities $(j, n)$.) Although constants of integration associated with reaction rates were discussed earlier, we consider them more explicitly here.

Figure 1 illustrates the effect of changing the value of $M_{1}$. If $M_{1}$ were very large, the high concentration of metal ions near the metal/oxide surface would oppose new ions from entering, whereas the high concentration of metal ions near the oxide/gas interface would encourage more ions to be deposited on the oxide/gas surface.

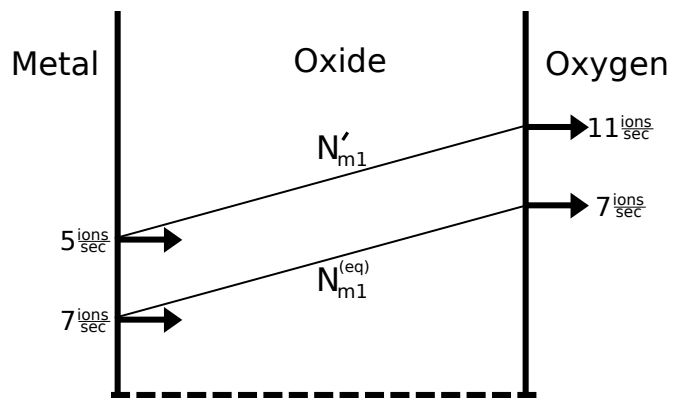

FIG. 1. The effect of the constant concentration $M_{1}$ of metal ions. Here $N_{m 1}^{\prime}$ has too high a value of $M_{1}$. Only the particular value of $M_{1}$ in $N_{m 1}^{(e q)}$ permits an equal rate of ions to enter and leave the oxide, here taken to be 7 ions per second.

Eventually, the number of metal ions in the bulk would be insufficient to maintain the high rate of ions exiting the oxide, and would drop to some equilibrium value. Thus, $M_{1}$ is determined by constraining the oxide to have no net ion-loading or ion-unloading in the bulk at order $n=1$. Note that $E_{1}$, which is proportional to (the parabolic growth rate coefficient), is also related to the surface reaction rates. In general, net surface reaction rates involve a Butler-Volmer equation, but not far from equilibrium (as in the Mott solution) they can be linearized in the differences of various electrochemical potentials. This will ensure that there is no net surface reaction rate in the limit of equilibrium.

From Gauss's Law at the surfaces 42,

$$
E_{1}(0)=\frac{\Sigma_{1}^{(0)}}{\epsilon}, \quad E_{1}(L)=-\frac{\Sigma_{1}^{(L)}}{\epsilon} .
$$

Since $E_{1}$ is uniform,

$$
\Sigma_{1}^{(0)}=-\Sigma_{1}^{(L)}=\epsilon E_{1} .
$$

For $\left|\nu_{a}\right|>>\left|\nu_{b}\right|$ (or equivalently in this case, $D_{a}>>$ $D_{b}$ ), Mott and Cabrera ${ }^{5}$ find for monovalent ions that

$$
J_{a 1}=-2 D_{b} \frac{\partial N_{a 1}}{\partial x} ;
$$

our results can be shown to be consistent with this.

\section{SOLUTION FOR $n=2$}

We here summarize the $n=2$ results. (For the explicit solution, see Appendix A.) Recall that all coefficients must be multiplied by $t$ to find the physical variables $(j, n, E)$. With the constant $M_{21}$ (in units $\mathrm{s} / \mathrm{m}^{2}$ ) determined by surface reaction rates (and thus linear in $E_{1}$ ), the second order flux density coefficients are

$$
J_{a 2}=Z J_{b 2}=-(1+Z)\left(\frac{D_{a} D_{b}}{Z D_{b}+D_{a}}\right) M_{21},
$$


and there is no net charge flux,

$$
J_{2}=Z e J_{b 2}-e J_{a 2}=0 .
$$

With the constants $M_{20}, P_{a 2}^{(+)}$and $P_{a 2}^{(-)}$(each in units of $\mathrm{s} / \mathrm{m}$ ) determined by surface reaction rates (and thus linear in $E_{1}$ ), the $n=2$ coefficients of the concentrations of electrons and ions are given by

$$
\begin{aligned}
N_{a 2}= & M_{20}+ \\
N_{b 2}=\frac{M_{20}}{Z}- & \frac{1}{Z} \frac{H \epsilon E_{1}^{2}}{V_{T} e}+\frac{M_{21}}{Z} x \\
& -P_{a 2}^{(+)} e^{x / l_{s}}-P_{a 2}^{(-)} e^{-x / l_{s}} .
\end{aligned}
$$

Here, $l_{s}$ is the screening length,

$$
l_{s}=\sqrt{\frac{V_{T} \epsilon}{(1+Z) n_{0} e}}=\sqrt{\frac{k_{B} T \epsilon}{(1+Z) n_{0} e^{2}}} .
$$

There is a net charge in the bulk, given by $\rho_{2} t^{-1}$, where

$$
\rho_{2}=P_{2}^{(+)} e^{x / l_{s}}+P_{2}^{(-)} e^{-x / l_{s}}-\frac{H \epsilon E_{1}^{2}}{V_{T}}
$$

and

$$
P_{2}^{(+)}=-(1+Z) e P_{a 2}^{(+)}, \quad P_{2}^{(-)}=-(1+Z) e P_{a 2}^{(-)} .
$$

Note that $\rho_{2}$ has, in addition to surface charge within a screening length of the two surfaces, a uniform charge density with sign determined by $e /\left(D_{a}-D_{b}\right)$ and independent of the sign of $E_{1}$ (or, equivalently, the direction of current flow). Since $D_{a} \gg D_{b}$ here, the term is positive. For holes and oxygen ions the carriers, we have $-e /\left(D_{a}-D_{b}\right)$, but $D_{b} \gg D_{a}$, so it is again positive. As found in previous work,, 7 this uniform charge density leads to a quadratic voltage profile within the bulk, not within a screening length of either surface.

The second order coefficient of the electric field is

$$
\begin{aligned}
E_{2}= & \frac{l_{s}}{\epsilon}\left(P_{2}^{(+)} e^{x / l_{s}}-P_{2}^{(-)} e^{-x / l_{s}}\right)-\frac{H E_{1}^{2}}{V_{T}} x \\
& +\frac{V_{T} M_{21}}{n_{0} H}-\frac{M_{1} E_{1}}{n_{0}} .
\end{aligned}
$$

The surface charge coefficients are given by

$$
\begin{aligned}
\Sigma_{2}^{(0)} & =l_{s}\left(P_{2}^{(+)}-P_{2}^{(-)}\right)+\frac{\epsilon V_{T} M_{21}}{n_{0} H}-\frac{\epsilon M_{1} E_{1}}{n_{0}} \\
\Sigma_{2}^{(L)} & =-l_{s}\left(P_{2}^{(+)} e^{L / l_{s}}-P_{2}^{(-)} e^{-L / l_{s}}\right)+\frac{\epsilon H E_{1}^{2}}{V_{T}} L \\
& -\frac{\epsilon V_{T} M_{21}}{n_{0} H}+\frac{\epsilon M_{1} E_{1}}{n_{0}} .
\end{aligned}
$$

We have verified that

$$
\Sigma_{2}^{(0)}+\Sigma_{2}^{(L)}+\int_{0}^{L} \rho_{2} d x=0
$$

so there is no net charge in the system.

\section{RATE OF GROWTH OF OXIDE LAYER}

The oxide layer grows as metal ions reach the $\mathrm{MO} / \mathrm{O}$ surface, and are taken into lattice positions to form a new oxide layer. Thus, the rate of growth of the oxide depends on the rate $j_{b}$ at which metal ions arrive, according to (4), or $d L / d t=\Omega j_{b}$. Including $n=3$ (see Appendix B we find the metal ion number flux (using Eqs. (53), (59) or (A17), and (B4),

$$
\begin{aligned}
j_{b}=( & \left.-\left(\frac{1+Z}{Z}\right) \frac{D_{a} D_{b}}{D_{b}-D_{a}} \frac{n_{0} E_{1}}{V_{T}}\right) t^{-1 / 2} \\
& +\left(-\left(\frac{1+Z}{Z}\right)\left(\frac{D_{a} D_{b}}{Z D_{b}+D_{a}}\right) M_{21}\right) t^{-1} \\
& +\left(\frac{H n_{0} E_{1}}{4 V_{T} Z} x^{2}+\frac{M_{1}}{2 Z} x+\frac{K_{3}}{Z}+\frac{\epsilon E_{1}}{2 Z e}\right) t^{-3 / 2}+\ldots,
\end{aligned}
$$

where $K_{3}$ is a constant of integration (units of flux density times $\mathrm{s}^{3 / 2}$ ) determined by interfacial reaction rates (and thus linear in $E_{1}$ ). Note that, if $D_{b}<D_{a}$ (as for ions relative to electrons) then the microscopics must give $E_{1}>0$ for a positive growth rate. Keeping only terms of second order, integration of 70 with respect to time gives

$$
L=\sqrt{2 A} t^{1 / 2}+B \ln t+\ldots
$$

where

$$
\begin{aligned}
& A=2\left(\frac{1+Z^{2}}{Z}\right)^{2}\left(\frac{D_{a} D_{b}}{D_{b}-D_{a}}\right)^{2} \frac{n_{0}^{2} E_{1}^{2} \Omega^{2}}{V_{T}^{2}} \\
& B=\frac{D_{a} D_{b}}{D_{a}+Z^{2} D_{b}}\left((1-Z) \frac{M_{1} E_{1}}{V_{T}}-\frac{1+Z^{2}}{Z} M_{21}\right) \Omega
\end{aligned}
$$

\section{SUMMARY AND CONCLUSION}

We have shown that the approach taken by Mott for parabolic growth of oxide films can be turned into a consistent asymptotic expansion, and we have explicitly given the form of the lowest three orders. Up to four integration constants appear at each order, related to the surface reaction rates. At higher order the bulk film is found to be charged, with a corresponding non-uniform current density.

The Appendices present the $n=2$ and $n=3$ solutions in detail, to show that the method can be used for any $n$ to find the fluxes, concentrations, surface charges and electric field. As a consequence one can have confidence that the Mott solution gives the leading term in the complete solution of the complete set of transport equations.

The most easily verifiable prediction from the viewpoint of experiment is the prediction that the first correction to the linear growth law is logarithmic. Because $\ln t$ is of order unity, data should be analyzed with an additional constant: $L=\sqrt{2 A} t+B \ln t+C+\ldots$ A sampling 
of the current literature ${ }^{12} \frac{16}{16}$ did not find enough precision to confirm the logarithmic form of the correction term.

We would like to thank Allan Jacobson for his comments and suggestions. This work was partially supported by the Department of Energy through grant DEFG02-06ER46278.

\section{Appendix A: Explicit Solution for $n=2$}

By the continuity equations $29 J_{a 2}$ and $J_{b 2}$ must be uniform, and by (36) they are related by

$$
J_{a 2}=Z J_{b 2} .
$$

For $n=2$, the Planck-Nernst equations from (44) and (45) are

$$
\begin{aligned}
& J_{a 2}+\frac{D_{a}}{V_{T}} n_{0} E_{2}+D_{a} \partial_{x} N_{a 2}=-\frac{D_{a}}{V_{T}} N_{a 1} E_{1}, \\
& J_{b 2}-\frac{D_{b}}{V_{T}} n_{0} E_{2}+D_{b} \partial_{x} N_{b 2}=\frac{Z D_{b}}{V_{T}} N_{b 1} E_{1} .
\end{aligned}
$$

Taking spatial derivatives of A2 and $\mathrm{A} 3$, and using Gauss's Law 41 and the uniformity of $J_{a 2}$ and $J_{b 2}$, yields

$$
\begin{aligned}
& \frac{D_{a} n_{0}}{V_{T} \epsilon} \rho_{2}+D_{a} \partial_{x}^{2} N_{a 2}=-\frac{D_{a}}{V_{T}} \partial_{x}\left(N_{a 1} E_{1}\right), \\
& -\frac{D_{b} n_{0}}{V_{T} \epsilon} \rho_{2}+D_{b} \partial_{x}^{2} N_{b 2}=\frac{Z D_{b}}{V_{T}} \partial_{x}\left(N_{b 1} E_{1}\right) .
\end{aligned}
$$

The right hand sides are found from 54);

$$
\begin{aligned}
& \frac{D_{a} n_{0}}{V_{T} \epsilon} \rho_{2}+D_{a} \partial_{x}^{2} N_{a 2}=-D_{a} \frac{H n_{0} E_{1}^{2}}{V_{T}^{2}}, \\
& -\frac{D_{b} n_{0}}{V_{T} \epsilon} \rho_{2}+D_{b} \partial_{x}^{2} N_{b 2}=D_{b} \frac{H n_{0} E_{1}^{2}}{V_{T}^{2}},
\end{aligned}
$$

where $H$ is defined in (55).

Subtracting (A6) multiplied by $1 / D_{a}$ from (A7) multiplied by $Z / D_{b}$ yields

$$
\partial_{x}^{2} \rho_{2}-(1+Z) \frac{n_{0} e}{V_{T} \epsilon} \rho_{2}=(1+Z) \frac{H n_{0} e E_{1}^{2}}{V_{T}^{2}} .
$$

The solution to this equation, with

$$
l_{s} \equiv \sqrt{\frac{V_{T} \epsilon}{(1+Z) n_{0} e}}=\sqrt{\frac{k_{B} T \epsilon}{(1+Z) n_{0} e^{2}}},
$$

and with new integration constants $P_{2}^{(+)}$and $P_{2}^{(-)}$, is

$$
\rho_{2}=P_{2}^{(+)} e^{x / l_{s}}+P_{2}^{(-)} e^{-x / l_{s}}-\frac{H \epsilon E_{1}^{2}}{V_{T}} .
$$

From A10 we infer that $N_{a 2}$ and $Z N_{b 2}$ are polynomials whose terms that are linear or higher are equal, and they may have different exponential terms.
Substituting $\rho_{2}$ from A10 into Gauss's Law 411, and integrating $\partial_{x} E_{2}$ yields

$$
E_{2}=\frac{l_{s}}{\epsilon}\left(P_{2}^{(+)} e^{x / l_{s}}-P_{2}^{(-)} e^{-x / l_{s}}\right)-\frac{H E_{1}^{2}}{V_{T}} x+F_{2},
$$

where $F_{2}$ is a new integration constant with units V-s/m.

By (54), $\left(N_{a 1}, N_{b 1}\right)$ are linear in $x$, so that the righthand-side of the Planck-Nernst equation A2 is linear in $x$. Moreover, the continuity equation (29) implies that $J_{a 2}$ is constant. Therefore by A11 for $E_{2}$, the PlanckNernst equation allows $\partial_{x} N_{a 2}$ to be linear, so $N_{a 2}$ can be quadratic in $x$. Moreover, the exponential terms in $\left(D_{a} / V_{T}\right) n_{0} E_{2}+D_{a} \partial_{x} N_{a 2}$ must cancel. Finally, from A10 any linear or quadratic terms in $N_{a 2}$ and $Z N_{b 2}$ must be equal. With $\left(M_{20}, M_{21}, M_{22}, P_{a 2}^{( \pm)}\right)$being new integration constants, we therefore conclude that the following form must hold:

$$
N_{a 2}=M_{20}+M_{21} x+M_{22} x^{2}+P_{a 2}^{(+)} e^{x / l_{s}}+P_{a 2}^{(-)} e^{-x / l_{s}} .
$$

Use of (31) for $n=2\left(\rho_{2}=e\left(Z N_{b 2}-N_{a 2}\right)\right)$, and $\rho_{2}$ of A10 gives

$$
\begin{aligned}
N_{b 2} & =\frac{M_{20}}{Z}-\frac{1}{Z} \frac{H \epsilon E_{1}^{2}}{V_{T} e}+\frac{M_{21}}{Z} x+\frac{M_{22}}{Z} x^{2} \\
& +\left(\frac{P_{2}^{(+)}+e P_{a 2}^{(+)}}{Z e}\right) e^{x / l_{s}}+\left(\frac{P_{2}^{(-)}+e P_{a 2}^{(-)}}{Z e}\right) e^{-x / l_{s}} .
\end{aligned}
$$

Addition of A2 divided by $D_{a}$ and (A3) divided by $D_{b}$ gives, with (A1) and 54),

$$
J_{a 2}\left(\frac{1}{D_{a}}+\frac{1}{Z D_{b}}\right)+\partial_{x}\left(N_{a 2}+N_{b 2}\right)=0 .
$$

Substitution for $N_{a 2}$ and $N_{b 2}$ from $\mathrm{A} 12$ and $\mathrm{A} 13$ allows us to solve for some of the constants,

$$
\begin{gathered}
M_{21}\left(1+\frac{1}{Z}\right)+2 M_{22}\left(1+\frac{1}{Z}\right) x \\
+\frac{1}{l_{s}}\left(P_{a 2}^{(+)}\left(1+\frac{1}{Z}\right)+\frac{P_{2}^{(+)}}{Z e}\right) e^{x / l_{s}} \\
-\frac{1}{l_{s}}\left(P_{a 2}^{(-)}\left(1+\frac{1}{Z}\right)+\frac{P_{2}^{(-)}}{Z e}\right) e^{-x / l_{s}} \\
=-J_{a 2}\left(\frac{1}{D_{a}}+\frac{1}{Z^{2} D_{b}}\right) .
\end{gathered}
$$

So, since $J_{a 2}$ is uniform, comparison of powers of $x$ yields the conditions

$$
\begin{aligned}
& P_{2}^{(+)}=-(1+Z) e P_{a 2}^{(+)}, \quad P_{2}^{(-)}=-(1+Z) e P_{a 2}^{(-)}, \\
& J_{a 2}=Z J_{b 2}=-(1+Z)\left(\frac{D_{a} D_{b}}{Z D_{b}+D_{a}}\right) M_{21}, \\
& M_{22}=0 .
\end{aligned}
$$


We may thus rewrite $\mathrm{A} 12$ and $\mathrm{A} 13$ as

$$
\begin{aligned}
& N_{a 2}= M_{20}+M_{21} x+P_{a 2}^{(+)} e^{x / l_{s}}+P_{a 2}^{(-)} e^{-x / l_{s}}, \\
& N_{b 2}=\frac{M_{20}}{Z}-\frac{1}{Z} \frac{H \epsilon E_{1}^{2}}{V_{T} e}+\frac{M_{21}}{Z} x \\
&-P_{a 2}^{(+)} e^{x / l_{s}}-P_{a 2}^{(-)} e^{-x / l_{s}} .
\end{aligned}
$$

Note that in A2 the exponential and linear coefficients already match, by construction. A new constraint, however, is found by comparing the constant terms,

$$
\begin{gathered}
-(1+Z)\left(\frac{D_{a} D_{b}}{Z^{2} D_{b}+D_{a}}\right) M_{21}+\frac{D_{a} n_{0}}{V_{T}} F_{2}+D_{a} M_{21} \\
=-\frac{D_{a} M_{1} E_{1}}{V_{T}}
\end{gathered}
$$

yielding the condition that

$$
F_{2}=\frac{V_{T} M_{21}}{n_{0} H}-\frac{M_{1} E_{1}}{n_{0}} .
$$

We are thus left with four independent constants of integration, which we take to be $M_{20}, M_{21}, P_{2}^{(+)}$, and $P_{2}^{(-)}$. The reaction rates for electrons and ions at each interface, needed to produce the correct ion fluxes, provide the 4 conditions necessary to solve for these constants.

For completeness we note that, from Gauss's Law at each surface 42,

$$
E_{2}(0)=\frac{\Sigma_{2}^{(0)}}{\epsilon}, \quad E_{2}(L)=-\frac{\Sigma_{2}^{(L)}}{\epsilon} .
$$

Then A11 and A22 yield

$$
\begin{aligned}
\Sigma_{2}^{(0)} & =l_{s}\left(P_{2}^{(+)}-P_{2}^{(-)}\right)+\frac{\epsilon V_{T} M_{21}}{n_{0} H}-\frac{\epsilon M_{1} E_{1}}{n_{0}}, \\
\Sigma_{2}^{(L)} & =-l_{s}\left(P_{2}^{(+)} e^{L / l_{s}}-P_{2}^{(-)} e^{-L / l_{s}}\right)+\frac{\epsilon H E_{1}^{2}}{V_{T}} L \\
& -\frac{\epsilon V_{T} M_{21}}{n_{0} H}+\frac{\epsilon M_{1} E_{1}}{n_{0}} .
\end{aligned}
$$

\section{Appendix B: Solution for $n=3$}

Recall that in order $n=3$, all coefficients must be multiplied by $t^{3 / 2}$ to find the physical variables $(j, n, E)$.

Using the $n=1$ coefficients for electron and metal ion number densities from (54) and the continuity equations from 30 for $n=3$,

$$
\partial_{x} J_{a 3}=\frac{H n_{0} E_{1}}{2 V_{T}} x+\frac{M_{1}}{2}, \quad \partial_{x} J_{b 3}=\frac{H n_{0} E_{1}}{2 V_{T} Z} x+\frac{M_{1}}{2 Z} x .
$$

Integration of these two equations gives two integration constants. However, these two constatnts are constrained by charge conservation across the interface at $x=0$ (37),

$$
\left.Z J_{b 3}\right|_{x=0}=\left.J_{a 3}\right|_{x=0}+\frac{\Sigma_{1}^{(0)}}{2 e},
$$

where $\Sigma_{1}^{(0)}$ is given by (57). Integration of the continuity equations (B1) then yields only a single new integration constant, which we call $K_{3}$ :

$$
\begin{aligned}
& J_{a 3}=\frac{H n_{0} E_{1}}{4 V_{T}} x^{2}+\frac{M_{1}}{2} x+K_{3}, \\
& J_{b 3}=\frac{H n_{0} E_{1}}{4 V_{T} Z} x^{2}+\frac{M_{1}}{2 Z} x+\frac{K_{3}}{Z}+\frac{\epsilon E_{1}}{2 Z e} .
\end{aligned}
$$

There is a uniform net electric charge flux (i.e., current density) at order $n=3$,

$$
J_{3}=e\left(Z J_{b 3}-J_{a 3}\right)=\frac{\epsilon E_{1}}{2},
$$

due to the discharge of the surfaces in order $n=1$.

For $n=3$, the Planck-Nernst equations from 44 and (45) are

$$
\begin{aligned}
J_{a 3}+\frac{D_{a}}{V_{T}} n_{0} E_{3}+D_{a} \partial_{x} N_{a 3} & =-\frac{D_{a}}{V_{T}}\left(N_{a 2} E_{1}+N_{a 1} E_{2}\right), \\
J_{b 3}-\frac{D_{b}}{V_{T}} n_{0} E_{3}+D_{b} \partial_{x} N_{b 3} & =\frac{Z D_{b}}{V_{T}}\left(N_{b 2} E_{1}+N_{b 1} E_{2}\right) .
\end{aligned}
$$

Taking spatial derivatives of (B6) and (B7), and using Gauss's Law 41,

$$
\begin{aligned}
\partial_{x} J_{a 3}+\frac{D_{a} n_{0}}{V_{T} \epsilon} \rho_{3}+ & D_{a} \partial_{x}^{2} N_{a 3} \\
& =-\frac{D_{a}}{V_{T}} \partial_{x}\left(N_{a 2} E_{1}+N_{a 1} E_{2}\right) \\
\partial_{x} J_{b 3}-\frac{D_{b} n_{0}}{V_{T} \epsilon} \rho_{3}+ & D_{b} \partial_{x}^{2} N_{b 3} \\
& =\frac{Z D_{b}}{V_{T}} \partial_{x}\left(N_{b 2} E_{1}+N_{b 1} E_{2}\right)
\end{aligned}
$$

Subtracting (B8 multiplied by $1 / D_{a}$ from B9 multiplied by $Z / D_{b}$ yields an equation for $\rho_{3}$,

$$
\begin{aligned}
& e\left(\frac{1}{D_{b}}-\frac{1}{D_{a}}\right) \partial_{x} J_{a 3}-(1+Z) \frac{n_{0} e}{V_{T} \epsilon} \rho_{3}+\partial_{x}^{2} \rho_{3}= \\
& \quad \frac{e E_{1}}{V_{T}}\left(\partial_{x} N_{a 2}+Z^{2} \partial_{x} N_{b 2}\right)+\frac{e}{V_{T}}\left(\partial_{x} N_{a 1}+Z^{2} \partial_{x} N_{b 1}\right) E_{2} \\
& \quad+\frac{e}{V_{T}}\left(N_{a 1}+Z^{2} N_{b 1}\right) \partial_{x} E_{2} .
\end{aligned}
$$

Substitution for $\left(N_{a 1}, N_{b 1}\right)$ from $(54),\left(N_{a 2}, N_{b 2}\right)$ from A19 and A20, $E_{2}$ from A11, and $J_{a 3}$ from (B3) yields 


$$
\begin{aligned}
\partial_{x}^{2} \rho_{3} & -\frac{1}{l_{s}^{2}} \Delta \rho_{3}= \\
& e\left(\frac{D_{b}-D_{a}}{D_{a} D_{b}}\right)\left(\frac{H n_{0} E_{1}}{2 V_{T}} x+\frac{M_{1}}{2}\right) \\
& +\frac{E_{1}}{V_{T}}\left((1+Z) e M_{21}-\left(\frac{1-Z^{2}}{1+Z}\right) \frac{P_{2}^{(+)}}{e l_{s}} e^{x / l_{s}}+\left(\frac{1-Z^{2}}{1+Z}\right) \frac{P_{2}^{(-)}}{e l_{s}} e^{-x / l_{s}}\right) \\
& +(1+Z) \frac{H n_{0} e E_{1}}{V_{T}^{2}}\left(F_{2}-\frac{H E_{1}^{2}}{V_{T}} x+\frac{l_{s} P_{2}^{(+)}}{\epsilon} e^{x / l_{s}}-\frac{l_{s} P_{2}^{(-)}}{\epsilon} e^{-x / l_{s}}\right) \\
& +(1+Z) \frac{e}{V_{T}}\left(\frac{H n_{0} E_{1}}{V_{T}} x+M_{1}\right)\left(-\frac{H E_{1}^{2}}{V_{T}}+\frac{P_{2}^{(+)}}{\epsilon} e^{x / l_{s}}+\frac{P_{2}^{(-)}}{\epsilon} e^{-x / l_{s}}\right) .
\end{aligned}
$$

The solution to this second order differential equation, with two new integration constants $\beta_{1}^{(+)}$and $\beta_{1}^{(-)}$, is

$$
\begin{aligned}
\rho_{3}= & \alpha_{1}+\alpha_{2} x+\beta_{1}^{(+)} e^{x / l_{s}}+\beta_{1}^{(-)} e^{-x / l_{s}}+\beta_{2}^{(+)} x e^{x / l_{s}} \\
& +\beta_{2}^{(-)} x e^{-x / l_{s}}+\beta_{3}^{(+)} x^{2} e^{x / l_{s}}+e \beta_{3}^{(-)} x^{2} e^{-x / l_{s}},
\end{aligned}
$$

where, with substitution of $F_{2}$ from A22,

$$
\begin{aligned}
& \alpha_{1}=-\left(\frac{D_{b}-D_{a}}{D_{a} D_{b}}\right) \frac{M_{1} e l_{s}^{2}}{2}-2 \frac{\epsilon E_{1} M_{21}}{n_{0}}+2 \frac{\epsilon H M_{1} E_{1}^{2}}{n_{0} V_{T}}, \\
& \alpha_{2}=-\left(\frac{D_{b}-D_{a}}{D_{a} D_{b}}\right) \frac{\epsilon H E_{1}}{2(1+Z)}+2 \frac{\epsilon H^{2} E_{1}^{3}}{V_{T}^{2}}, \\
& \beta_{2}^{(+)}=\left(-\left(\frac{1-Z^{2}}{1+Z}\right) \frac{E_{1}}{2 V_{T}}+\frac{H E_{1}}{4 V_{T}}+\frac{M_{1}}{2 n_{0} l_{s}}\right) P_{2}^{(+)} \\
& \beta_{2}^{(-)}=\left(-\left(\frac{1-Z^{2}}{1+Z}\right) \frac{E_{1}}{2 V_{T}}+\frac{H E_{1}}{4 V_{T}}-\frac{M_{1}}{2 n_{0} l_{s}}\right) P_{2}^{(-)} \\
& \beta_{3}^{(+)}=\frac{H E_{1}}{4 V_{T} l_{s}} P_{2}^{(+)}, \quad \beta_{3}^{(-)}=-\frac{H E_{1}}{4 V_{T} l_{s}} P_{2}^{(-)} .
\end{aligned}
$$

From (B12) we infer that $N_{a 3}$ and $Z N_{b 3}$ may include polynomials whose terms that are quadratic and higher are equal, and may include exponential terms that differ.

Substituting $\rho_{3}$ from (B12) into Gauss's Law (41), and integrating $\partial_{x} E_{3}$ gives

$$
\begin{aligned}
E_{3} & =F_{3}+\frac{\alpha_{1}}{\epsilon} x+\frac{\alpha_{2}}{2 \epsilon} x^{2} \\
& +\frac{l_{s}}{\epsilon}\left(\beta_{1}^{(+)}-l_{s} \beta_{2}^{(+)}+2 l_{s}^{2} \beta_{3}^{(+)}\right) e^{x / l_{s}} \\
& -\frac{l_{s}}{\epsilon}\left(\beta_{1}^{(-)}+l_{s} \beta_{2}^{(-)}+2 l_{s}^{2} \beta_{3}^{(-)}\right) e^{-x / l_{s}} \\
& +\frac{l_{s}}{\epsilon}\left(\beta_{2}^{(+)}-2 l_{s} \beta_{3}^{(+)}\right) x e^{x / l_{s}} \\
& -\frac{l_{s}}{\epsilon}\left(\beta_{2}^{(-)}+2 l_{s} \beta_{3}^{(-)}\right) x e^{-x / l_{s}} \\
& +\frac{l_{s}}{\epsilon} \beta_{3}^{(+)} x^{2} e^{x / l_{s}}-\frac{l_{s}}{\epsilon} \beta_{3}^{(-)} x^{2} e^{-x / l_{s}},
\end{aligned}
$$

where $F_{3}$ is a new integration constant, with units $\mathrm{V}$ $\mathrm{s}^{3 / 2} / \mathrm{m}$.

Substitution of the coefficients $N_{a 1}$ from (54), $N_{a 2}$ from A12, $E_{2}$ from (A11), $J_{a 3}$ from (B3), and $E_{3}$ from (B18) into the $n=3$ Planck-Nernst equation for electrons (B6) yields 


$$
\begin{aligned}
\partial_{x} & N_{a 3}=-\left(\frac{M_{20} E_{1}}{V_{T}}+\frac{M_{1} F_{2}}{V_{T}}+\frac{n_{0} F_{3}}{V_{T}}+\frac{K_{3}}{D_{a}}\right) \\
& +\left(\frac{H M_{1} E_{1}^{2}}{V_{T}^{2}}-\frac{M_{21} E_{1}}{V_{T}}-\frac{H n_{0} F_{2} E_{1}}{V_{T}^{2}}-\frac{n_{0} \alpha_{1}}{\epsilon V_{T}}-\frac{M_{1}}{2 D_{a}}\right) x \\
& +\left(\frac{H^{2} n_{0} E_{1}^{3}}{V_{T}^{3}}-\frac{n_{0} \alpha_{2}}{2 \epsilon V_{T}}-\frac{H n_{0} E_{1}}{4 D_{a} V_{T}}\right) x^{2} \\
& +\left(\frac{E_{1} P_{2}^{(+)}}{(1+Z) e V_{T}}-\frac{l_{s} M_{1} P_{2}^{(+)}}{\epsilon V_{T}}-\frac{n_{0} l_{s}}{\epsilon V_{T}}\left(\beta_{1}^{(+)}-l_{s} \beta_{2}^{(+)}+2 l_{s}^{2} \beta_{3}^{(+)}\right)\right) e^{x / l_{s}} \\
& +\left(\frac{E_{1} P_{2}^{(-)}}{(1+Z) e V_{T}}+\frac{l_{s} M_{1} P_{2}^{(-)}}{\epsilon V_{T}}+\frac{n_{0} l_{s}}{\epsilon V_{T}}\left(\beta_{1}^{(-)}+l_{s} \beta_{2}^{(-)}+2 l_{s}^{2} \beta_{3}^{(-)}\right)\right) e^{-x / l_{s}} \\
& +\left(-\frac{n_{0} l_{s} H E_{1} P_{2}^{(+)}}{\epsilon V_{T}^{2}}-\frac{n_{0} l_{s}}{\epsilon V_{T}}\left(\beta_{2}^{(+)}-2 l_{s} \beta_{3}^{(+)}\right)\right) x e^{x / l_{s}} \\
& +\left(\frac{n_{0} l_{s} H E_{1} P_{2}^{(-)}}{\epsilon V_{T}^{2}}+\frac{n_{0} l_{s}}{\epsilon V_{T}}\left(\beta_{2}^{(-)}+2 l_{s} \beta_{3}^{(-)}\right)\right) x e^{-x / l_{s}} \\
& -\frac{n_{0} l_{s}}{\epsilon V_{T}} \beta_{3}^{(+)} x^{2} e^{x / l_{s}}+\frac{n_{0} l_{s}}{\epsilon V_{T}} \beta_{3}^{(-)} x^{2} e^{-x / l_{s}} .
\end{aligned}
$$

The solution to this first order differential equation, with one new integration constant $M_{30}$, is where

$$
\begin{aligned}
M_{31}= & -\left(\frac{M_{20} E_{1}}{V_{T}}+\frac{M_{1} F_{2}}{V_{T}}+\frac{n_{0} F_{3}}{V_{T}}+\frac{K_{3}}{D_{a}}\right), \\
M_{32}= & \frac{1}{2}\left(\frac{H M_{1} E_{1}^{2}}{V_{T}^{2}}-\frac{M_{21} E_{1}}{V_{T}}\right. \\
\left.\quad-\frac{H n_{0} F_{2} E_{1}}{V_{T}^{2}}-\frac{n_{0} \alpha_{1}}{\epsilon V_{T}}-\frac{M_{1}}{2 D_{a}}\right), & (\mathrm{B} 21) \\
M_{33}= & \frac{1}{3}\left(\frac{H^{2} n_{0} E_{1}^{3}}{V_{T}^{3}}-\frac{n_{0} \alpha_{2}}{2 \epsilon V_{T}}-\frac{H n_{0} E_{1}}{4 D_{a} V_{T}}\right), \\
\gamma_{1}^{(+)}= & \left(\frac{(H+1) l_{s} E_{1}}{V_{T}}-\frac{M_{1}}{n_{0}}\right) \frac{P_{2}^{(+)}}{(1+Z) e} \\
& -\frac{1}{(1+Z) e}\left(\beta_{1}^{(+)}-2 l_{s} \beta_{2}^{(+)}+6 l_{s}^{2} \beta_{3}^{(+)}\right), \\
\gamma_{1}^{(-)}= & -\left(\frac{M_{1}}{n_{0}}+\frac{(H+1) l_{s} E_{1}}{V_{T}}\right) \frac{P_{2}^{(-)}}{(1+Z) e} \\
& -\frac{1}{(1+Z) e}\left(\beta_{1}^{(-)}+2 l_{s} \beta_{2}^{(-)}+6 l_{s}^{2} \beta_{3}^{(-)}\right), \quad(\mathrm{B} 25) \\
\gamma_{2}^{(+)}= & -\frac{H E_{1} P_{2}^{(+)}}{(1+Z) e V_{T}}-\frac{1}{(1+Z) e}\left(\beta_{2}^{(+)}-4 l_{s} \beta_{3}^{(+)}\right), \\
\gamma_{2}^{(-)}= & -\frac{H E_{1} P_{2}^{(-)}}{(1+Z) e V_{T}}-\frac{1}{(1+Z) e}\left(\beta_{2}^{(-)}+4 l_{s} \beta_{3}^{(-)}\right), \\
\gamma_{3}^{(+)}= & -\frac{\beta_{3}^{(+)}}{(1+Z) e}, \quad \gamma_{3}^{(-)}=-\frac{\beta_{3}^{(-)}}{(1+Z) e} .
\end{aligned}
$$

Use of (31) for $n=3\left(\rho_{3}=e\left(Z N_{b 3}-N_{a 3}\right)\right), \rho_{3}$ from 
(B12), and $N_{a 3}$ from (B20) gives

$$
\begin{aligned}
N_{b 3} & =\left(\frac{\alpha_{1}+e M_{30}}{Z e}\right)+\left(\frac{\alpha_{2}+e M_{31}}{Z e}\right) x \\
& +\left(\frac{M_{32}}{Z}\right) x^{2}+\left(\frac{M_{33}}{Z}\right) x^{3}+\left(\frac{\beta_{1}^{(+)}+e \gamma_{1}^{(+)}}{Z e}\right) e^{x / l_{s}} \\
& +\left(\frac{\beta_{1}^{(-)}+e \gamma_{1}^{(-)}}{Z e}\right) e^{-x / l_{s}}+\left(\frac{\beta_{2}^{(+)}+e \gamma_{2}^{(+)}}{Z e}\right) x e^{x / l_{s}} \\
& +\left(\frac{\beta_{2}^{(-)}+e \gamma_{2}^{(-)}}{Z e}\right) x e^{-x / l_{s}}+\left(\frac{\beta_{3}^{(+)}+e \gamma_{3}^{(+)}}{Z e}\right) x^{2} e^{x / l_{s}} \\
& +\left(\frac{\beta_{3}^{(-)}+e \gamma_{3}^{(-)}}{Z e}\right) x^{2} e^{-x / l_{s}} .
\end{aligned}
$$

We now have $J_{a 3}, J_{b 3}, E_{3}, N_{a 3}$ and $N_{b 3}$ with five constants of integration, $K_{3}, \beta_{1}^{(+)}, \beta_{1}^{(-)}, F_{3}$, and $M_{30}$. To reduce this from five to four, we add (B6) divided by $D_{a}$ and (B7) divided by $D_{b}$,

$$
\frac{J_{a 3}}{D_{a}}+\frac{J_{b 3}}{D_{b}}+\partial_{x}\left(N_{a 3}+N_{b 3}\right)=\frac{1}{V_{T}}\left(\frac{\rho_{2} E_{1}}{e}+\frac{\rho_{1} E_{2}}{e}\right) .
$$

As for $n=2$, in $\mathrm{B} 30$ the exponential, quadratic, and linear terms match, by construction. A new constraint, however, is found by comparing the constant terms,

$$
\frac{K_{3}}{D_{a}}+\frac{K_{3}}{Z D_{b}}+\frac{\epsilon E_{1}}{2 Z e D_{b}}+M_{31}+\frac{\alpha_{2}+e M_{31}}{Z e}=-\frac{H \epsilon E_{1}^{3}}{V_{T}^{2} e} .
$$

Substituting $M_{31}$ from (B21) yields

$$
\begin{aligned}
F_{3}= & \left(\frac{D_{b}-D_{a}}{(1+Z) D_{a} D_{b}}\right) K_{3}-\frac{M_{20} E_{1}}{n_{0}}-\frac{M_{1} F_{2}}{n_{0}} \\
& -\left(\frac{1}{(1+Z) e}\right)\left(\frac{Z H \epsilon E_{1}^{3}}{V_{T}^{2}}+\frac{\epsilon E_{1}}{2 D_{b}}+\alpha_{2}\right),
\end{aligned}
$$

a relation between integration constants $F_{3}$ and $K_{3}$.

We are thus left with four independent constants of integration, which we take to be $K_{3}, M_{30}, \beta_{1}^{(+)}$, and $\beta_{1}^{(-)}$. The reaction rates for electrons and ions at each interface, needed to produce the correct ion fluxes, provide the four conditions necessary to solve for these constants.

For completeness, we note that from Gauss's Law at each surface 42,

Then, (B18) yields

$$
E_{3}(0)=\frac{\Sigma_{3}^{(0)}}{\epsilon}, \quad E_{3}(L)=-\frac{\Sigma_{3}^{(L)}}{\epsilon} .
$$

$$
\begin{aligned}
& \Sigma_{3}^{(0)}=\epsilon F_{3}+l_{s}\left(\beta_{1}^{(+)}-l_{s} \beta_{2}^{(+)}+2 l_{s}^{2} \beta_{3}^{(+)}\right) \\
& \quad-l_{s}\left(\beta_{1}^{(-)}+l_{s} \beta_{2}^{(-)}+2 l_{s}^{2} \beta_{3}^{(-)}\right) \\
& \Sigma_{3}^{(L)}=-\epsilon F_{3}-\alpha_{1} L-\frac{\alpha_{2}}{2} L^{2} \\
& \quad-l_{s}\left(\beta_{1}^{(+)}-l_{s} \beta_{2}^{(+)}+2 l_{s}^{2} \beta_{3}^{(+)}\right) e^{L / l_{s}} \\
& \quad+l_{s}\left(\beta_{1}^{(-)}+l_{s} \beta_{2}^{(-)}+2 l_{s}^{2} \beta_{3}^{(-)}\right) e^{-L / l_{s}} \\
& \quad-l_{s}\left(\beta_{2}^{(+)}-2 l_{s} \beta_{3}^{(+)}\right) L e^{L / l_{s}} \\
& \quad+l_{s}\left(\beta_{2}^{(-)}+2 l_{s} \beta_{3}^{(-)}\right) L e^{-L / l_{s}} \\
& \quad-l_{s} \beta_{3}^{(+)} L^{2} e^{L / l_{s}}+l_{s} \beta_{3}^{(-)} L^{2} e^{-L / l_{s}} .
\end{aligned}
$$

We have verified that

$$
\Sigma_{3}^{(0)}+\Sigma_{3}^{(L)}+\int_{0}^{L} \rho_{3} d x=0,
$$

so there is no net charge in the system.
1 N.F. Mott. Trans. Faraday Soc., 35:1175, 1939.

2 N.F. Mott. Trans. Faraday Soc., 35:472, 1940.

3 N.F. Mott. Trans. Faraday Soc., 43:429, 1947.

${ }^{4}$ G. Z. Tammann. anorg. allg. Chem., 111:78, 1920.

${ }^{5}$ N. Cabrera and N. F. Mott. Rep. Prog. Phys., 12:163, 1949.

6 C. Wagner. Atom Movements. Am. Soc. for Metals, Cleveland, Ohio, 1951.

7 W. M. Saslow. Phys. Rev. Lett., 76:4849, 1996.

8 Y. Yang and W. M. Saslow. J. Chem. Phys., 109:10331, 1998.
9 D. R. Crow. Principles and Applications of Electrochemistry, 4th ed. Blackie Academic \& Professional, 1994.

10 J. M. Rubi and S. Kjelstrup. J. Phys. Chem. B, 107:13471, 2003.

11 J. Fleig. Phys. Chem. Chem. Phys., 7:2027, 2005.

12 A. A. Dravnieks. J. Phys. Chem., 55:540, 1951.

13 M. Martin. Thin Solid Films, 250:61, 1994.

14 S. K. Lahiri. Microelectronics Journal, 29:335, 1998.

15 L. P. H. Jeurgens et al. J. Appl. Phys., 92:1649, 2002.

16 C. Zhong et al. Appl. Phys. A, 90:263, 2008. 